\title{
Charge-induced formation of linear Au clusters on thin MgO films: Scanning tunneling microscopy and density-functional theory study
}

\author{
Violetta Simic-Milosevic ${ }^{1}$, Markus Heyde ${ }^{1}$, Xiao Lin ${ }^{1}$, Thomas König ${ }^{1}$, Hans-Peter Rust ${ }^{1}$, \\ Martin Sterrer ${ }^{1}$, Thomas Risse ${ }^{1}$, Niklas Nilius ${ }^{1, *}$ Hans-Joachim Freund ${ }^{1}$ \\ Livia Giordano ${ }^{2}$, Gianfranco Pacchioni ${ }^{2, *}$ \\ ${ }^{1}$ Fritz-Haber-Institut der Max-Planck-Gesellschaft, Department of Chemical Physics, \\ Faradayweg 4-6, D-14195 Berlin, Germany \\ ${ }^{2}$ Dipartimento di Scienza dei Materiali, Università di Milano-Bicocca, Milano, Italy
}

Gold deposition onto ultra-thin $\mathrm{MgO}$ films on $\mathrm{Ag}(001)$ results in the formation of linear $\mathrm{Au}$ clusters, as revealed from a combined scanning tunneling microscopy and density functional theory study. The equilibrium structure of small Au clusters containing 6-7 atoms is therefore different on thin-film and bulk $\mathrm{MgO}(001)$, but also deviates from the shape of the respective gasphase clusters. The peculiar one-dimensional growth regime of gold is stimulated by an electron transfer from the $\mathrm{MgO} / \mathrm{Ag}$ interface to the deposited Au clusters, resulting in singly and doubly charged cluster anions. Only for larger atom numbers, the formation of compact, twodimensional clusters prevails on the MgO thin films.

\section{PACS:}

68.43.Fg (Adsorbate structure), 68.47.Gh (Oxide surfaces),

68.37.Ef (Scanning tunneling microscopy) 71.15.Mb (Density functional theory)

*Corresponding authors: nilius@fhi-berlin.mpg.de, gianfranco.pacchioni@unimib.it 


\section{Introduction}

The electronic and geometric structure of gold clusters deposited on oxide surfaces is attracting an increasing interest in both catalysis and surface science communities. ${ }^{1}$ The surprising discovery that, once in the form of nano-particles, even this inert metal becomes an active catalyst, ${ }^{2,3}$ has stimulated many attempts to elucidate the reasons for the enhanced reactivity. So far, the chemical properties of nano-structured gold have been related to the nature and pretreatment of the oxide support, ${ }^{4}$ as well as to the size and shape of the deposits. ${ }^{5}$ Also the charge state of Au species is assumed to play a decisive role. While some studies suggest $\mathrm{Au}^{\delta+}$ (cationic gold) to be the active phase, ${ }^{6,7,8}$ in other cases, negatively charged clusters, both in the gas-phase ${ }^{9}$ and on oxide supports ${ }^{10,11}$ were found to be more active than their positively charged or neutral counterparts. In a widely accepted picture, the excess charges facilitate the breaking of bonds in molecules adsorbed on the Au deposits and hence lower the activation barrier for surface reactions.

Anionic gold species can be produced in various ways. On bulk-like oxides, e.g. MgO and $\mathrm{TiO}_{2}$, charging occurs due to electron transfer from electron-rich surface defects, e.g. oxygen vacancies (F centers), as demonstrated by DFT calculations and IRAS using CO as a probe. ${ }^{12,13,14}$ An alternative charging mechanism prevails on ultra-thin oxide films $\left(\mathrm{MgO}, \mathrm{Al}_{10} \mathrm{O}_{13}\right)$ grown on metal supports. ${ }^{15,16}$ Here, electrons are transferred into initially unoccupied affinity levels of the adsorbate via tunneling from the metal support. The electron flow is facilitated by a reduction of the metal work-function that often accompanies the growth of thin oxide films on metal surfaces, as shown for MgO films on $\mathrm{Ag}(001)$ and $\mathrm{Mo}(001) .{ }^{17,18,19}$ The charging of electronegative species on thin oxide films therefore follows the oxidation model for metals developed by Cabrera and Mott. ${ }^{20}$

Negative charging of individual Au adatoms and clusters has been demonstrated, both experimentally and theoretically, for a few oxide films. Single Au atoms deposited on MgO thin 
films on $\operatorname{Ag}(001)$ self-assemble into a hexagonal array of adatoms, indicating the presence of Coulomb repulsion between the negatively charged species. ${ }^{21}$ With increasing exposure, twodimensional $\mathrm{Au}$ islands form on the oxide film, ${ }^{22}$ although Vollmer-Weber growth is typically observed for Au on bulk MgO. The deviating growth regime provides additional evidence for the accumulation of extra electrons in the Au aggregates, enabling enhanced polarization interactions and thus stronger binding to the oxide surface. ${ }^{23,24}$ Recent non-spin polarized DFT studies have shown that also Au clusters containing up to six atoms become negatively charged on $\mathrm{MgO} / \mathrm{Mo}(001)$ films and consequently assume different conformations than on bulk $\mathrm{MgO}(001) \cdot{ }^{24,25}$ On alumina thin

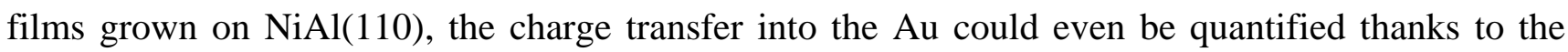
unique shape of the ad-clusters. ${ }^{26}$ The Au atoms self-assemble into one-dimensional (1D) chains on this surface and develop distinct quantum well states due to the overlap of the $A u$ 6s orbitals in neighboring chain atoms. By comparing the electron filling of those states with the number of $6 \mathrm{~s}$ electrons in the initial gas-phase clusters, the amount of charge transfer was determined to range between one and three electrons depending on the chain length. ${ }^{27}$ Although not proven experimentally, the formation of negatively charged Au species is expected to increase the reactivity of thin-film systems, opening a promising way to tune the catalytic performance of such composite materials. $^{28,29}$

In this paper, we report on a combined scanning tunneling microscopy (STM) and density functional theory (DFT) study of the properties of ultra-small Au clusters formed on ultra-thin MgO films on $\mathrm{Ag}(001)$. The clusters spontaneously adopt one-dimensional (1D) shapes upon $\mathrm{Au}$ deposition, but also arrange in a linear way during manipulation experiments performed with the STM tip. The distinct structural properties are confirmed by DFT calculations and traced back to a 
negative charging of the ad-chains. Au on $\mathrm{MgO} / \mathrm{Ag}(001)$ behaves therefore similar to the alumina on NiAl(110) case, providing another example for the "unusual" nature of gold on thin oxide films.

\section{Experimental and computational details}

The experiments were performed in a custom-built, Eigler style, ultrahigh-vacuum STM operating at $5 \mathrm{~K}^{30}$ The Ag(001) sample was cleaned by repeated cycles of $\mathrm{Ar}^{+}$sputtering (500 V, $10 \mathrm{~mA}$ ) and annealing to $700 \mathrm{~K}$. MgO films of 2-3 ML thickness were prepared by reactive Mg deposition at 570 $\mathrm{K}$ in an oxygen ambiance of $1 \times 10^{-6}$ mbar. Single Au atoms were deposited from a high purity wire (99.95\%) wrapped with a tungsten filament onto the sample surface held at $100 \mathrm{~K}$. The deposition temperature was therefore higher than in previous experiments to enforce the development of $\mathrm{Au}$ equilibrium structures on the oxide surface..$^{13,21,31}$ The coverage was adjusted between 0.01 and 0.02 ML. Whereas at low coverage, Au monomers are the predominant surface species, a large collection of differently shaped aggregates appear at higher Au exposure.

The DFT calculations were performed in the generalized gradient approximation (PW-91 exchange-correlation functional $)^{32}$ as implemented in the VASP code. ${ }^{33,34}$ A plane wave basis set (400 eV energy cutoff) and the projected augmented wave method (PAW) ${ }^{35}$ was used for the core electrons. The substrate was modeled by four $\mathrm{Ag}(001)$ planes, adding two MgO layers to one side of the slab. The residual dipoles perpendicular to the surface were eliminated with a specific dipole correction scheme. To account for the differently sized Au clusters, $(3 \times 3),(4 \times 4)$ and $(5 \times 5)$ supercells were constructed. Only for $\mathrm{Au}_{5}$ and $\mathrm{Au}_{6}$ chains, the use of a (4×7) cell became necessary. The atoms in the super-cells were relaxed until the atomic forces fell below $0.01 \mathrm{eV} / \AA$. The electronic properties were determined by Brillouin-zone integrations on a $(4 \times 4 \times 1)$ Monkhorst-Pack grid ${ }^{36}$ for the $(3 \times 3)$ cell, while an equally-spaced k-point sampling was applied to larger unit cells. Except for 
the largest cell, calculations were carried out in a spin-polarized scheme in order to account for the possible presence of unpaired electrons in the valence shell of the Au clusters.

\section{III.A Experimental results}

Figure 1 shows a typical STM topographic image of a 2 ML thick MgO film on $\mathrm{Ag}(001)$ after deposition of 0.02 ML of Au. The majority species are round protrusion of $0.8 \AA \AA$ height, which are assigned to single Au adatoms. Also differently shaped Au aggregates are occasionally found in the images, for instance small triangular-shaped clusters (see square in Fig. 1). In the focus of this paper are, however, chain-like Au aggregates, which are frequently observed on the MgO surface (arrows in Fig. 1). Most of the chains align with the MgO [100] direction, which is the crystallographic orientation where $\mathrm{Mg}$ and $\mathrm{O}$ atoms alternate on the surface. However, also [110] oriented Au chains are found on the thin MgO film (circle in Fig. 1).

Seven different chains could be discriminated in the images on the basis of their length (Fig. 2). The largest one is approximately $22 \AA$ long, when measuring the distance between the two terminal points on which the topographic chain height reaches 50\% of its maximum value (FWHM). For each shorter chain, the length reduces by 2.5-3.0 $\AA$, getting down to the Au monomer that is characterized by a spherical shape and $6 \AA$ apparent diameter. According to this length estimate, the longest chain on the MgO surface corresponds to an $\mathrm{Au}$ heptamers $\left(\mathrm{Au}_{7}\right)$, while the most frequent ones are trimer and tetramer chains. In the bias window available for surface imaging $(-1.0$ to +1.0 V), single atoms and Au chains have similar apparent heights of $0.8 \AA$. The only exception is a small, spherical Au species that is approximately 40\% higher than the monomer (Fig. 2B). ${ }^{31}$

The electronic properties of linear Au clusters were investigated with differential conductance $(\mathrm{dI} / \mathrm{dV})$ spectroscopy. Independent of the chain length, the spectra did not reveal peaks in the 
conductance and were similar to the ones taken on the bare MgO film (Fig. 3). Apparently, no initial/final states are available for tunneling into/out-of the ad-structures, as also suggested by their uniform apparent height in STM imaging. Only in $\mathrm{d} / \mathrm{dV}$ maps taken at negative bias, a small increase in the signal becomes visible at both sides of longer chains (Fig. 3A,B). It should be mentioned at this point that conductance measurements were hampered by the small bias window available for spectroscopy $( \pm 1.0 \mathrm{~V})$. Applying bias values outside this range usually caused the alteration of the Au aggregates, as manifested by their desorption, motion or dissociation.

The high susceptibility of oxide-supported Au atoms to the injection of energetic electrons was, however, exploited to manipulate their binding configuration and spatial arrangement on the surface. For this purpose, the tip was positioned above a selected Au species (a single atom or an aggregate) and the voltage was increased above the manipulation threshold (usually to $2.0 \mathrm{~V}$ ) with enabled feedback loop. A sudden decrease of the tip height manifested the success of the manipulation experiment and a change of the adsorbate structure. In the majority of cases, the manipulation procedure led to the displacement of Au atoms in a large area below the tip position. The required atom mobility was hereby generated by the tip-induced electric field or alternatively by the temporal occupation of mixed $\mathrm{Au}-\mathrm{MgO}$ states with anti-bonding character. In few examples, $\mathrm{Au}$ monomers also aggregated upon electron injection (Fig. 4). Surprisingly, also these artificial clusters adopted 1D shapes, manifesting the thermodynamic preference for the Au chain formation on thin MgO films. The strong trend for a linear arrangement of $\mathrm{Au}$ atoms on $\mathrm{MgO} / \mathrm{Ag}(001)$ and the properties of the resulting ad-chains shall be discussed in the light of DFT calculations in the following. 


\section{III.B Theoretical results}

As discussed in earlier publications, ${ }^{15,21,24} \mathrm{Au}$ atoms are able to form strong bonds to a 2 ML thick MgO film on $\mathrm{Ag}(001)$. The large increase in binding energy with respect to bulk $\mathrm{MgO}$ (2.3 eV versus $1.0 \mathrm{eV}$ ) originates from a negative charging of the adatom via electron transfer from the Ag support. The $\mathrm{Au}^{-}$species is able to lock into the Madelung field of the oxide film and induces a polaronic distortion of the $\mathrm{MgO}$ that enhances the polarization interaction with the anionic species. The preferred $\mathrm{Au}$ binding sites are identified as the $\mathrm{Mg}^{2+}$ top site and the hollow site in the oxide surface, in contrast to bulk MgO where binding to the oxygen anions is clearly favored. The energetic preference of cationic over anionic sites is, however, small $(0.25 \mathrm{eV})$ for the thin $\mathrm{MgO}$ film, leading to a flat potential energy surface for adsorption. ${ }^{15}$

The smallest $\mathrm{Au}$ aggregate, the dimer, forms in two different configurations on the $\mathrm{MgO} /$ $\mathrm{Ag}(001)$ surface (Fig. 5). ${ }^{31}$ In its most stable configuration, the $\mathrm{Au}_{2}$ has an upright geometry with the lower atom attached to a surface oxygen ion $\left(\mathrm{r}_{\mathrm{Au}-\mathrm{Au}}=252 \mathrm{pm}, \mathrm{r}_{\mathrm{Au}-\mathrm{O}}=217 \mathrm{pm}\right)$. The upright dimer is neutral and has a closed shell electronic structure, in analogy to Au dimers on bulk MgO(001). ${ }^{37}$ Furthermore, different flat-lying $\mathrm{Au}_{2}$ species are stable on the thin film, which are either bound to two $\mathrm{Mg}^{2+}$ ions or to two hollow sites and align with the MgO[100] or [110] direction. In all cases, the flat $\mathrm{Au}_{2}$ is slightly higher in energy $(0.34 \mathrm{eV})$ than its upright counterpart and carries an additional electron (-0.8e), as deduced from a Bader charge analysis. A closer look to the charge density difference reveals that the electron is transferred from the $\mathrm{MgO} / \mathrm{Ag}$ interface region. The negative charging of the flat dimer is compatible with two additional observations, namely the enlarged $\mathrm{Au}-\mathrm{Au}$ distance of $264 \mathrm{pm}$ (similar to an gas-phase $\mathrm{Au}_{2}{ }^{-}$) and the strong polaronic distortion of the $\mathrm{MgO}$ lattice around the anionic species. Since flat-lying $\mathrm{Au}_{2}{ }^{-}$cannot be stabilized on 
bulk MgO, the emergence of this species is directly related to the presence of the metal support below the oxide film and the associated charge transfer.

Despite this strong stabilization effect of the $\mathrm{MgO} / \mathrm{Ag}(001)$ support, the neutral and upright $\mathrm{Au}_{2}$ is the lowest energy isomer and not the flat-lying species. This unexpected result is rationalized by the fact that the extra electron in the flat $\mathrm{Au}_{2}{ }^{-}$occupies an anti-bonding $6 s-6 s$ hybrid state and therefore weakens the inter-atomic interaction. The lower Au-Au binding energy is not compensated by the stronger adhesion to the surface and the flat-lying configuration remains energetically less favorable. Interestingly, the flat and upright dimers are nearly iso-energetic on MgO films on $\mathrm{Mo}(001)$, reflecting the even stronger impact of the Mo support on the Au-MgO interaction. ${ }^{25}$ For both substrates, the thermodynamic stability of the flat-lying $\mathrm{Au}_{2}{ }^{-}$species decreases with increasing film thickness, demonstrating the diminishing influence of the metal support on the adhesion energy.

To characterize the nucleation behavior of dimers and larger Au aggregates on the MgO thin film, the attachment energy $E_{b}$ of a single adatom to an existing $\mathrm{Au}_{\mathrm{n}-1}$ cluster is calculated via:

$$
E_{b}\left(A u_{n}\right)=-E\left(A u_{n} / M g O\right)-E(M g O)+E\left(A u_{n-1} / M g O\right)+E\left(A u_{1} / M g O\right)
$$

The formation energy for Au dimers on the $2 \mathrm{ML} \mathrm{MgO/Ag(001)} \mathrm{film} \mathrm{is} \mathrm{determined} \mathrm{with} 0.63 \mathrm{eV}$ and $0.29 \mathrm{eV}$ for the vertical and flat configuration, respectively (2.1 eV on bulk MgO) (Fig. 6). The low values reflect the rather small adhesion of neutral and singly charged dimers with respect to two negatively charged and hence strongly bound adatoms. During the aggregation process, also the Coulomb repulsion between the two $\mathrm{Au}^{-}$species needs to be overcome and one electron has to be transferred back into the substrate, introducing an additional barrier for dimer formation. However, as the attachment energy is still positive, Au dimers are expected to form spontaneously on the MgO thin film. 
In agreement with the DFT calculations, Au dimers in both, the flat-lying and the upright configuration are unambiguously identified in STM images of the surface (Fig. 2B,C). Whereas the flat-lying species shows up as ellipsoidal feature with $9 \AA$ apparent length and $0.8 \AA$ height, the vertical one is a round protrusion of slightly larger height $(1.1 \AA) .{ }^{31}$ In accordance to the theoretical predictions, the ellipsoidal structures assigned to flat dimers are less frequently observed on thin MgO films than the upright species, reflecting their lower thermodynamic stability and formation energy.

Adding a third $\mathrm{Au}$ atom to a vertical $\mathrm{Au}_{2}$ dimer on $2 \mathrm{ML} \mathrm{MgO/Ag(001)} \mathrm{leads} \mathrm{to} \mathrm{the}$ formation of a neutral, upright Au triangle that is similar to the lowest-energy isomer on bulk $\mathrm{MgO}(001)$. However, a flat-lying $\mathrm{Au}_{3}$ chain is by far more stable on the thin film than the upright triangle, in contrast to the dimer case (Fig. 5). In the lowest energy configuration, the three atoms occupy neighboring MgO hollow sites in [100] direction. Two other geometries, where the trimer axis points along the [110] direction or the $\mathrm{Au}$ atoms sit atop $\mathrm{Mg}^{2+}$ sites are almost iso-energetic (energy difference below $0.1 \mathrm{eV}$ ). The $\mathrm{Au}_{3}$ chain carries one extra electron similar to the flat $\mathrm{Au}$ dimer, as proven by the Bader analysis, the polaronic distortion of the oxide lattice and the absence of unpaired electrons in the trimer ground state. The clear preference for the formation of charged flat-lying $\mathrm{Au}_{3}$ species is compatible with the high electron affinity of the trimer with respect to the dimer (3.7 versus $2.0 \mathrm{eV}) .{ }^{38}$ The stability of the $\mathrm{Au}_{3}{ }^{-}$chain on the thin film support is also reflected by the high formation energy of $0.94 \mathrm{eV}$, which exceeds the one for triangular shaped clusters on bulk $\mathrm{MgO}(001)$ by $60 \%\left(\mathrm{E}_{\mathrm{b}}=0.58 \mathrm{eV}\right.$, Fig. 6$)$. Attempts to locate other triangular isomers that lie flat on the surface failed, as all structures spontaneously relaxed into the 1D configuration.

A linear cluster oriented along $\mathrm{MgO}[100]$ or [110] also represents the most stable configuration for the Au tetramer on the thin magnesia film (Fig. 5). The 1D structure is preferred by 
$0.8 \mathrm{eV}$ over the standing rhombus, which is the lowest-energy isomer on bulk $\mathrm{MgO}(001)$. Also other possible configurations, such as a Y-shaped structure, were tested and found to be less stable by about $0.7 \mathrm{eV}$ with respect to the chain. In contrast, the $\mathrm{Y}$-shaped configuration has been proposed as the most stable isomer on Mo-supported MgO films, most likely due to the different nature of the MgO/Mo(001) interface. ${ }^{24,25}$ It should be noted yet that the linear chain was not considered in that study. Similar to the dimer and trimer case, the linear arrangement of atoms in $\mathrm{Au}_{4}$ clusters is driven by a charge transfer from the metal support. However, even spin polarized calculations of the tetramer chain yield a diamagnetic ground state, although the gas-phase cluster is a closed shell system and the extra electron should therefore induce a magnetic moment. A plausible explanation for the non-magnetic ground state is the transfer of two electrons from the $\mathrm{Ag}(001)$ support into the gold cluster, which is indeed confirmed by the Bader analysis (Fig. 5). The presence of two extra electrons also explains the one-dimensional shape of the cluster, as only in this configuration the additional charge is optimally delocalized and the internal electrostatic repulsion is minimized. The tendency to separate the extra electrons is also reflected by the fact that the two terminal chain atoms carry higher fractional charges than the central ones (Fig. 5). As the charge distribution in a compact rhombus is less efficient, the total energy of $2 \mathrm{D}$ isomers is higher despite the larger number of internal $\mathrm{Au}-\mathrm{Au}$ bonds. The $\mathrm{Au}_{4}$ on $\mathrm{MgO}$ thin films is not the only example for a doubly charged tetramer chain as a similar species has recently been found on alumina thin films. ${ }^{27}$

Although formation of linear $\mathrm{Au}$ clusters on $\mathrm{MgO} / \mathrm{Ag}(001)$ is favored for small atom numbers, the $1 \mathrm{D}$ growth has to pass into a $2 \mathrm{D}$ regime with increasing coverage, according to recent STM results. ${ }^{22}$ Also DFT calculations revealed that gold clusters of 8 to 20 atoms deposited on ultrathin MgO films assume flat 2D structures, instead of the linear shapes obtained here. ${ }^{23}$ It is thus instructive to analyze the size range, in which the transition between 1D and 2D growth occurs. For 
this purpose, the energy of $\mathrm{Au}_{5}$ and $\mathrm{Au}_{6}$ clusters on $\mathrm{MgO} / \mathrm{Ag}(001)$ and bulk $\mathrm{MgO}(001)$ supports is determined for several linear and flat-lying 2D configurations (Fig. 5). On bulk MgO, none of these structures is adopted and Au clusters assume a flat shape with the plane normal to the surface. ${ }^{39,40}$ On the thin film, on the other hand, 1D and flat-lying 2D isomers are found to be iso-energetic for the $\mathrm{Au}_{5}$ species (Fig. 5). While the 2D aggregate is singly charged, the linear configuration accommodates two extra-electrons that are mainly localized at the two terminal atoms (Fig. 5). The formation energy of an $\mathrm{Au}_{5}$ chain is with $0.2 \mathrm{eV}$ rather small, which relates to the energy cost to form a magnetic pentamer with a half-filled electronic state located at the Fermi level. Still, the linear $\mathrm{Au}_{5}$ chain forms spontaneously on the surface, but might represent a critical size where 1D cluster growth on $\mathrm{MgO} / \mathrm{Ag}(001)$ breaks down.

This assumption is confirmed by the DFT results for different $\mathrm{Au}_{6}$ isomers, being the largest cluster considered in this work. The Au hexamer adsorbs preferentially in a flat 2D geometry (Fig. 5), which is $0.3 \mathrm{eV}$ lower in energy than the $1 \mathrm{D}$ chain. Both conformers accommodate two extra electrons transferred from the metal/oxide interface. The efficiency to distribute the excess electrons becomes comparable in the two aggregates, as the terminal atoms holding large fractions of the charge are sufficiently separated in both cases (Fig. 5). However, the flat structure enables the formation of three additional $\mathrm{Au}-\mathrm{Au}$ bonds, which makes this isomer finally more stable than the chain and indicates the crossover to the 2D growth regime.

\section{Discussion}

The calculated preference for the formation of ultra-small Au clusters with linear shapes is unambiguously confirmed by the experimental observations. After deposition of $0.02 \mathrm{ML}$ Au onto $\mathrm{MgO} / \mathrm{Ag}(001)$ thin films, small Au chains are the predominant ad-structures besides individual 
adatoms. The analysis of STM images taken in several experimental runs reveals that trimer and tetramer chains are the most frequently found linear species on the surface. ${ }^{41}$ This observation is in agreement with the high formation energies of linear $\mathrm{Au}_{3}\left(\mathrm{E}_{\mathrm{b}}=0.94\right)$ and $\mathrm{Au}_{4}$ clusters $(0.8 \mathrm{eV})$ (Fig. 6). In contrast, the formation energy exhibits a pronounced minimum for $\mathrm{Au}_{5}$ chains, whose synthesis out of an $\mathrm{Au}_{4}$ cluster is equally probable as its reorganization into a compact $\mathrm{Au}_{5}$ cluster. In accordance, $\mathrm{Au}_{5}$ chains are hardly found on the surface. The fact that even longer chains are experimentally observed only emphasizes the meta-stable character of the pentamer chain and its high tendency to transform into linear or $2 \mathrm{D} \mathrm{Au}_{6}$ clusters via atom attachment.

The longest chains identified in the STM images are $\mathrm{Au}_{6}$ (2 exemplars) and $\mathrm{Au}_{7}$ species (1 exemplar), which already coexist with various 2D clusters of approximately the same size. The experimentally determined transition between $1 \mathrm{D}$ and 2D growth therefore occurs for 6-7 atoms in the Au aggregates. This number is in good agreement with the DFT results (5-6 atoms) and the small discrepancy might be related to kinetic effects of the chain formation that are not accounted for in theory. Interestingly, the same critical size for the cross-over in the growth regime has earlier been determined for $\mathrm{Au}$ aggregates on alumina/NiAl(110), ${ }^{27}$ suggesting a certain universality of the underlying effect: The change from $1 \mathrm{D}$ to 2D growth mode occurs when the energy surplus from delocalizing the excess charges is outweighed by the formation of additional Au-Au bonds in compact 2D and 3D aggregates.

Finally, the absence of peaks in the $\mathrm{dI} / \mathrm{dV}$ spectra of Au chains on thin MgO films shall be commented, in particular because a set of eigen-states was detected for similar ad-chains on alumina/NiAl. ${ }^{27}$ The main difference between both systems is the increased sensitivity of MgOsupported Au chains against electron/hole injection from the tip, which limits the available bias range for spectroscopy to $\pm 1 \mathrm{~V}$ (compared to $\pm 3 \mathrm{~V}$ for alumina/NiAl). The large stability of alumina 
supported aggregates at high bias combines two effects: ${ }^{27}$ Due to an unusual interaction mechanism that involves the cleavage of an $\mathrm{Al}-\mathrm{O}$ bond, Au atoms are able to bind stronger to alumina than to magnesia films. Furthermore, the ad-chains form a surface complex with the top-most Al atoms of the alumina film, which enables a better delocalization of the charges injected from the tip and reduces the temporal Coulomb repulsion within the ad-chain and to the oxide support. The only detectable feature in dI/dV maps of linear Au clusters on $2 \mathrm{ML}$ MgO on $\mathrm{Ag}(001)$ are the pronounced end-states located at both sides of the chains (Fig. 3). This increase in occupied state density might be related to the localization of excess electrons at the terminal chain atoms that has been deduced from the DFT calculations (Fig. 5). The presence of zero-dimensional end-states might therefore provide another hint for the negative charging of the Au chains on the MgO film.

\section{Conclusion}

Employing STM and DFT, we have demonstrate that ultra-small gold clusters formed on thin MgO films on $\mathrm{Ag}(001)$ adopt a linear geometry. This particular cluster shape has no counterpart in the gasphase or on bulk MgO(001) surfaces, but is the consequence of the interaction between the adclusters and the metal support below the oxide film. The driving force for the chain formation is a charge transfer through the thin film into the Au clusters that satisfies the strong electronegative character of gold. The excess charges, amounting to two extra electrons for $\mathrm{Au}_{4}$ to $\mathrm{Au}_{6}$ chains, enable a strong polarization interaction with the oxide support; but simultaneously enforce the linear arrangement of the atoms in order to minimize the internal Coulomb repulsion. As the growth of Au chains is also observed on alumina thin films, it might reflect a general trend in gold nucleation on those oxide films that support formation of anionic Au species. 
It would be highly desirable to verify the charged nature of the Au clusters via an independent experimental technique. Several observables are sensitive to the charge state of adsorbates, for instance the energy position of core levels in XPS, the vibrational signature of probe molecules such as $\mathrm{CO}$, and the work function change induced by charged ad-species. However, the detection of the charge state of Au clusters via spatially averaging methods might be extremely difficult due to their small abundance on the MgO film and is beyond the scope of this paper.

\section{Acknowledgments}

The work has been supported by the COST Action D41 “Inorganic oxides: surfaces and interfaces”. Part of the computing time was provided by the Barcelona Supercomputing Center (BSC-CNS).

\section{Figures}

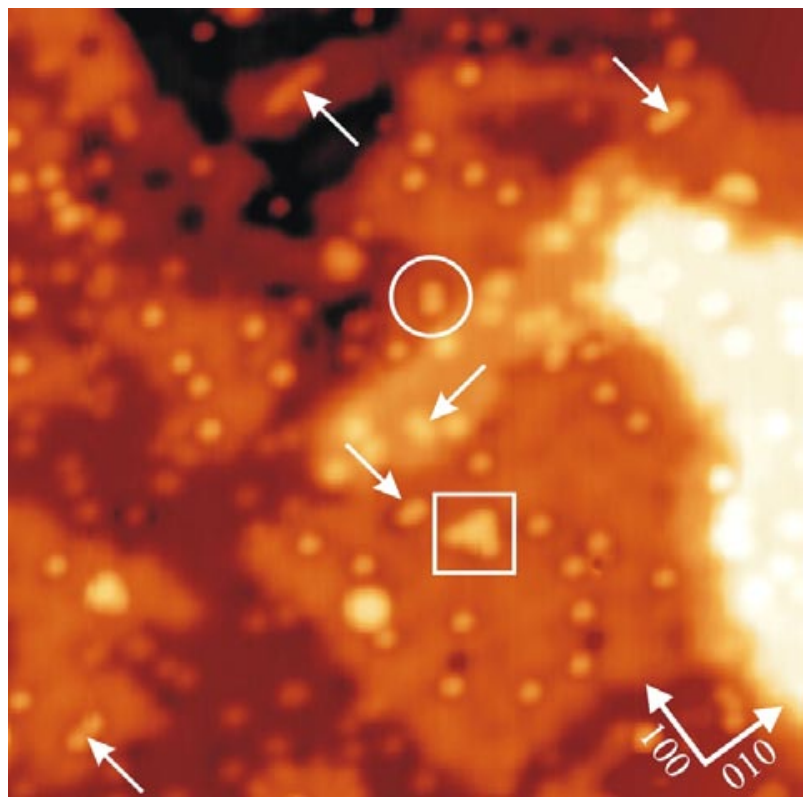

Fig. 1

(Color online): STM topographic image of Au adatoms and clusters on a 2 ML thick MgO film on $\mathrm{Ag}(001)\left(-0.4 \mathrm{~V}, 19 \times 19 \mathrm{~nm}^{2}\right)$. The arrows mark [100] and [010] oriented Au chains, while the circle depicts a chain in [110] direction. The squares frame two triangular 2D clusters. 

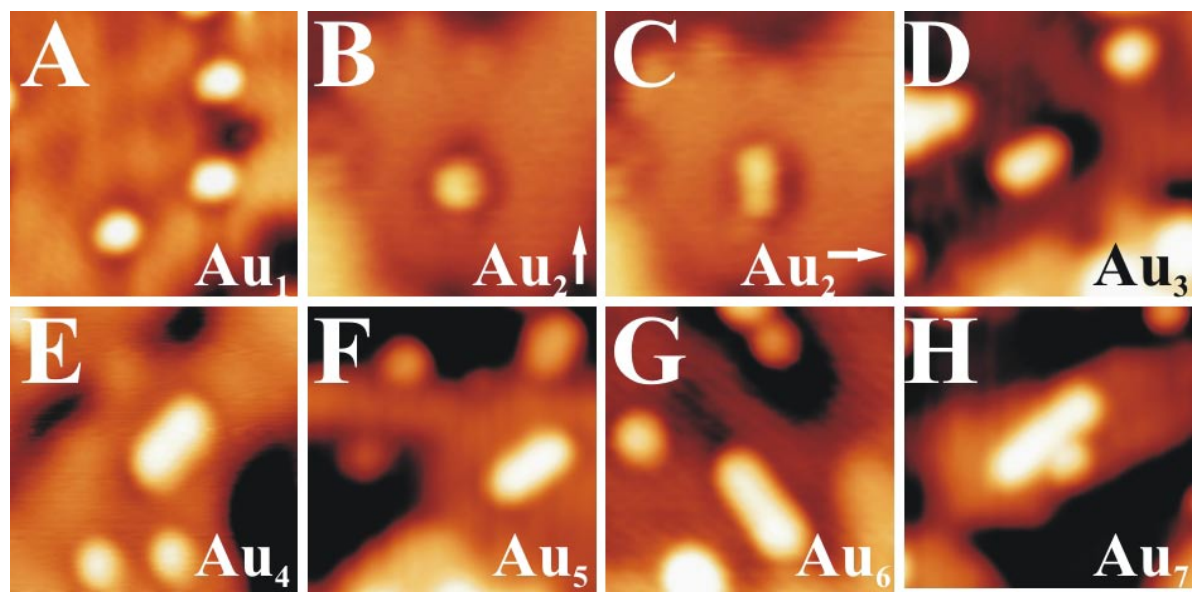

Fig. 2

(Color online): STM topographic images of (A) Au monomers, (B,C) upright and flat Au dimers and (D-H) Au chains containing three to seven atoms on $\mathrm{MgO} / \mathrm{Ag}(001)\left(-0.4 \mathrm{~V}, 5 \times 5 \mathrm{~nm}^{2}\right)$.

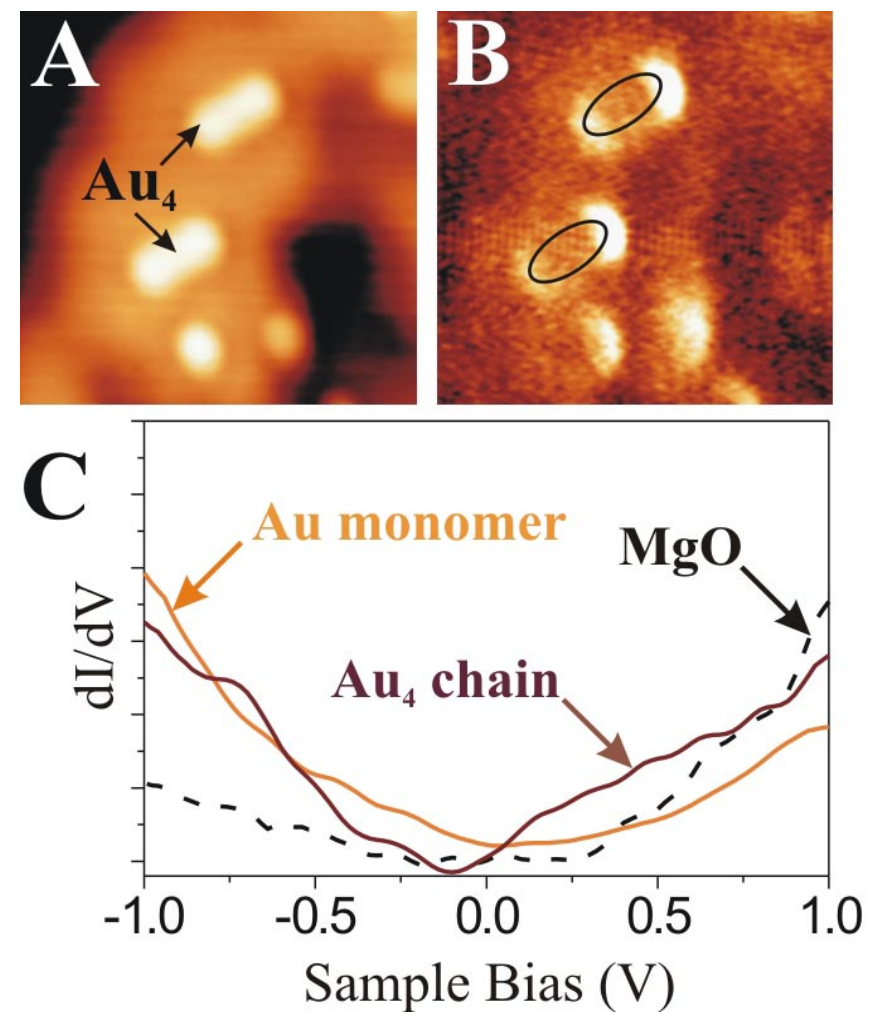

Fig. 3

(Color online): (A) Topographic and (B) differential conductance image of two $\mathrm{Au}_{4}$ chains on $\mathrm{MgO} /$ $\mathrm{Ag}(001)\left(-0.4 \mathrm{~V}, 6.5 \times 6.5 \mathrm{~nm}^{2}\right)$. (C) $\mathrm{dI} / \mathrm{dV}$ spectra of a monomer and an $\mathrm{Au}_{4}$ chain (setpoint bias +0.8 V). Apart from the end-states, no dI/dV features are visible in spectroscopy within the accessible bias window. 


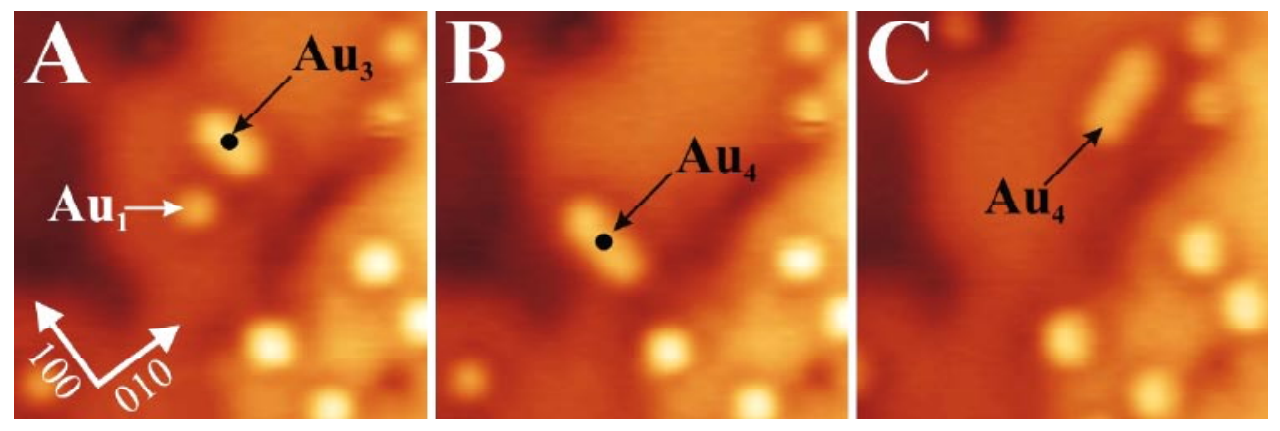

Fig. 4.

(Color online): (A) STM image showing a trimer and monomers on the MgO film. A +2V bias pulse was applied to the trimer at the position marked by the black dot, creating a tetramer chain (B). The same manipulation procedure was repeated for the tetramer (C), inducing the diffusion of the adchain.
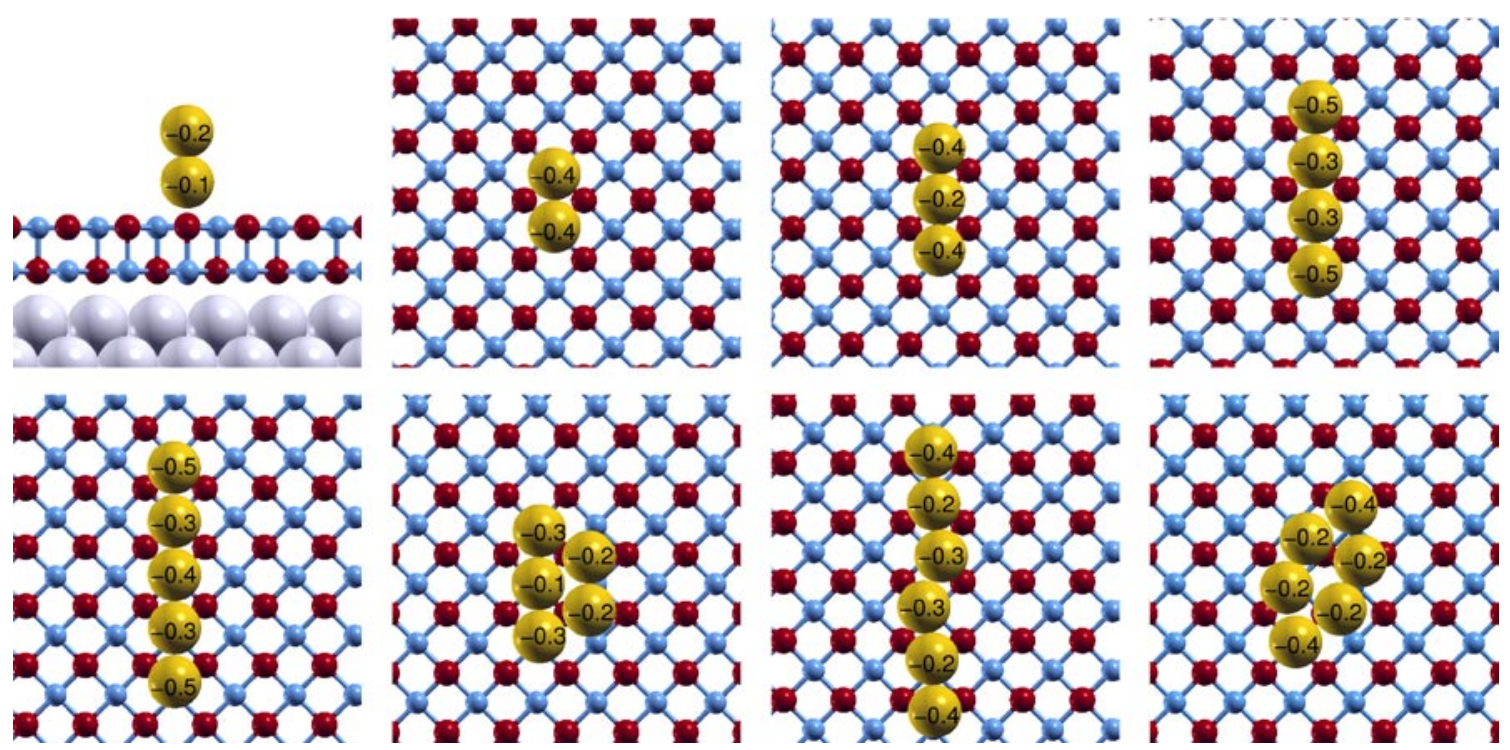

Fig. 5.

(Color online): Most stable $\mathrm{Au}_{\mathrm{n}}$ isomers $(\mathrm{n}=2-6)$ on $2 \mathrm{ML} \mathrm{MgO/Ag(001).} \mathrm{Red} \mathrm{spheres:} \mathrm{O}$ atoms, blue spheres: Mg atoms, yellow spheres: Au atoms. Bader charges are displayed on the corresponding Au atoms (in $|\mathrm{e}|)$. 


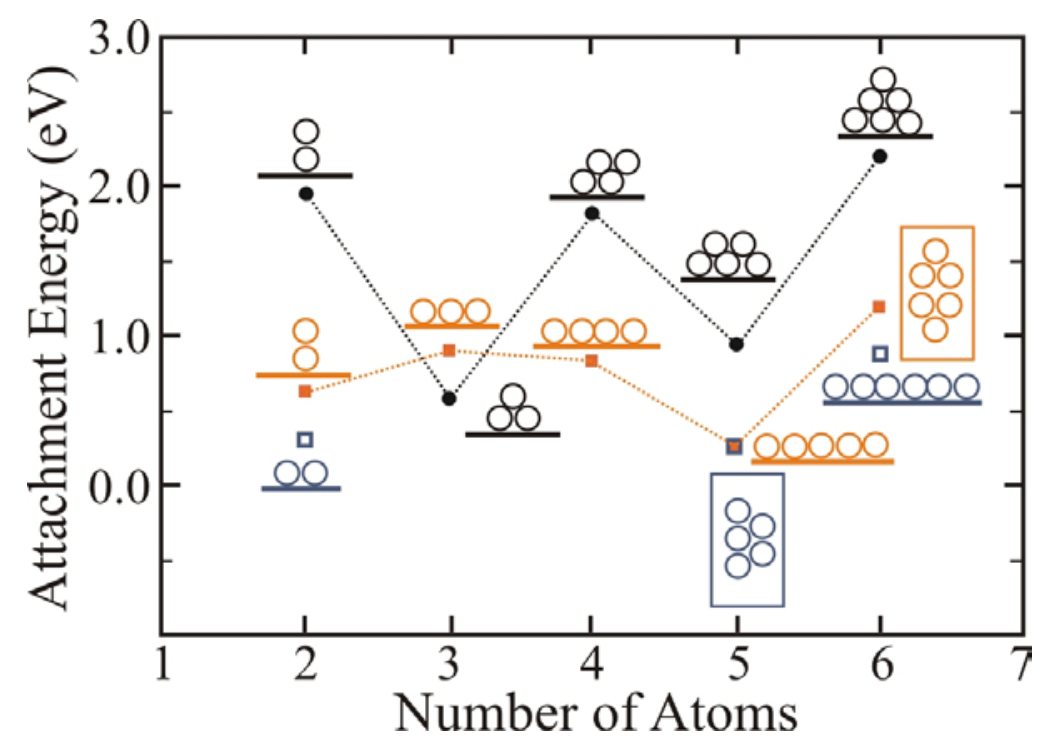

Fig. 6.

(Color online): Calculated atom attachment energies to form the Au clusters shown in the insets, either for bulk $\mathrm{MgO}(001)$ (black circles) or for $2 \mathrm{ML} \mathrm{MgO}$ on $\mathrm{Ag}(001)$ (orange squares). The blue squares depict the second most stable isomers on the thin film. While pronounced odd-even oscillations in the attachment energy are visible on bulk $\mathrm{MgO}$, such behavior is suppressed on the thin film. This relates to the removal of most open-shell configurations by electron transfer trough the $\mathrm{MgO}$ film (e.g. for $\mathrm{Au}_{3}$ ) and to the screening of unpaired electrons by the support for the remaining magnetic clusters (e.g. $\left.\mathrm{Au}_{5}\right)$. Attachment energies on the thin film are also lower than on bulk MgO, as each aggregation step implies the bond formation between two charged species.

\footnotetext{
${ }^{1}$ M. Chen, D. W. Goodman, Acc. Chem. Res. 39, 739 (2006).

${ }^{2}$ M. Haruta, T. Kobayashi, H. Sano, N. Yamada, Chem. Lett. 405 (1987).

${ }^{3}$ M. Haruta, Cat. Tech. 6, 102 (2002).

${ }^{4}$ S. Arrii, F. Morfin, A. J. Renouprez, J. L. Rousset, J. Am. Chem. Soc. 126, 1199 (2004).

${ }^{5}$ N. Lopez, J. Norskov, J. Am. Chem. Soc. 124, 11262 (2002).

${ }^{6}$ J. Guzman, B.C. Gates, Nano Lett 1, 689 (2001).

${ }^{7}$ Z.-P. Liu, S. J. Jenkins, D. A. King, Phys. Rev. Lett. 94, 196102 (2005).

${ }^{8}$ J. G. Wang, B. Hammer, Phys. Rev. Lett. 97, 136107 (2006).

${ }^{9}$ L. D. Socaciu, J. Hagen, T. M. Bernhardt, L. Wöste, U. Heiz, H. Häkkinen, U. Landman, J. Am. Chem. Soc. 125, 10437 (2003).

${ }^{10}$ B. Yoon, H. Häkkinen, U. Landman, A. S. Wörz, J. Antonietti, S. Abbet, J. Judai, U. Heiz, Science, 307, 403 (2005).

${ }^{11}$ L. M. Molina, B. Hammer, J. Catal. 233, 399 (2005).

${ }^{12}$ D. Matthey, J. W. Wang, S. Wendt, J. Matthiesen, R. Schaub, E. Laegsgaard, B. Hammer, F. Besenbacher, Science 315 (2007) 1692.

${ }^{13}$ M. Sterrer, M. Yulikov, E. Fishbach, M. Heyde, H.-P. Rust, G. Pacchioni, T. Risse, H.-J. Freund, Angew. Chemie Int. Ed. 45, 2630 (2006).

${ }^{14}$ A. S. Wörz, U. Heiz, F. Cinquini, G. Pacchioni, J. Phys. Chem. B 109, 18418 (2005).

${ }^{15}$ G. Pacchioni, L. Giordano, M. Baistrocchi, Phys. Rev. Lett. 94, 226104 (2005).

${ }^{16}$ L. Giordano, G. Pacchioni, J. Goniakowski, N. Nilius, E. D. L. Rienks, H.-J. Freund, Phys. Rev. Lett. 101, 026102 (2008).
} 
${ }^{17}$ L. Giordano, F. Cinquini, G. Pacchioni, Phys. Rev. B 73, 045414 (2005).

${ }^{18}$ H.-C. Ploigt, C. Brun, M. Pivetta, F. Patthey, W.-D. Schneider, Phys. Rev. B 76, 195404 (2007).

${ }^{19}$ H. M. Benia, N. Nilius, H.-J. Freund, Surf. Sci. 601, L55 (2007).

${ }^{20}$ N. Cabrera, N. F. Mott, Rep. Prog. Phys. 12, 163 (1948-1949).

${ }^{21}$ M. Sterrer, T. Risse, U. Martinez Pozzoni, L. Giordano, M. Heyde, H.-P. Rust, G. Pacchioni, H.-J. Freund, Phys. Rev. Lett. 98, 906017 (2007).

${ }^{22}$ M. Sterrer, T. Risse, M. Heyde, H.-P. Rust, H.-J. Freund, Phys. Rev. Lett. 98, 206103 (2007).

${ }^{23}$ D. Ricci, A. Bongiorno, G. Pacchioni, U. Landman, Phys. Rev. Lett. 97 , 036106 (2006).

${ }^{24}$ P. Frondelius, H. Häkkinen, K. Honkala, Phys. Rev. B 76, 073406 (2007).

${ }^{25}$ P. Frondelius, H. Häkkinen, K. Honkala, New J. Phys. 9, 339 (2007)

${ }^{26}$ M. Kulawik, N. Nilius, H.-J. Freund, Phys. Rev. Lett. 96, 036103 (2006).

${ }^{27}$ N. Nilius, V. Ganduglia-Pirovano, V. Bradzova, M. Kulawik, J. Sauer, H.-J. Freund, Phys. Rev. Lett. 100, 096802 (2008).

${ }^{28}$ C. Zhang, B. Yoon, U. Landman, J. Am. Chem. Soc. 129, 2228 (2007).

${ }^{29}$ H.-J. Freund, Surf. Sci. 601, 1438 (2007).

${ }^{30}$ M. Heyde, M. Kulawik, H.-P. Rust, H.-J. Freund, Rev. Sci. Instrum. 75, 2446 (2004).

${ }^{31}$ V. Simic-Milosevic, M. Heyde, N. Nilius, T. König, H.-P. Rust, M. Sterrer, T. Risse, H.-J. Freund, L. Giordano, G. Pacchioni, J. Amer. Chem. Soc. 130, 7814 (2008).

32 J. P. Perdew, J. A. Chevary, S. H. Vosko, K. A. Jackson, M. R. Pederson, D. J. Singh and C. Fiolhais, Phys. Rev. B 46, 6671 (1992).

${ }^{33}$ G. Kresse and J. Hafner, Phys. Rev. B 47, 558 (1993).

${ }^{34}$ G. Kresse and J. Furthmüller, Phys. Rev. B 54, 11169 (1996).

${ }^{35}$ P. E. Blöchl, Phys. Rev. B 50, 17953 (1994).

${ }^{36}$ H. J. Monkhorst and J. D. Pack, Phys. Rev. B 13, 5188 (1976).

${ }^{37}$ A. Del Vitto, G. Pacchioni, F. Delbecq, P. Sautet, J. Phys. Chem B 109, 8040 (2005).

${ }^{38}$ K. J. Taylor, C. L. Pettiette-Hall, O. Cheshnovsky, R. E. Smalley, J. Chem. Phys. 96, 3319 (1992).

${ }^{39}$ G. Barcaro, A. Fortunelli, J. Chem. Theory Comput. 1, 972, (2005).

${ }^{40}$ L. M. Molina, J. A. Alonso, J. Phys. Chem. C 111, 6668 (2007).

${ }^{41}$ This is in contrast to experiments where Au deposition has been performed at $10 \mathrm{~K} .{ }^{31}$ There, Au dimers are the dominant species next to the monomers, manifesting the presence of a kinetic barrier for the formation of longer chains. 